\title{
Acute Respiratory Decompensation Requiring Intubation in Pregnant Women with SARS-CoV-2 (COVID-19)
}

Jenna S. Silverstein, MD ${ }^{1,2}$ Meghana A. Limaye, $\mathrm{MD}^{3,4}$ Sara G. Brubaker, MD, MPH ${ }^{3,4}$ Ashley S. Roman, MD, MPH ${ }^{3}$ Judita Bautista, $\mathrm{MD}^{1,2}$ Judith Chervenak, $\mathrm{MD}^{3,4}$ Adam J. Ratner, MD, MPH ${ }^{5}$ Philip M. Sommer, MD ${ }^{6}$ Nicole M. Roselli, MD, MBA ${ }^{1,2}$ Charlisa D. Gibson, $\mathrm{MD}^{7}$ David Ellenberg, $\mathrm{MD}^{7}$ Christina A. Penfield, MD, MPH ${ }^{3,4}$

1 Department of Obstetrics and Gynecology, New York University Langone Medical Center, New York, New York

2 Department of Obstetrics and Gynecology, Bellevue Hospital Center, New York, New York

${ }^{3}$ Division of Maternal Fetal Medicine, Department of Obstetrics and Gynecology, New York University Langone Medical Center, New York, New York

${ }^{4}$ Division of Maternal Fetal Medicine, Department of Obstetrics and Gynecology, Bellevue Hospital Center, New York, New York

${ }^{5}$ Division of Pediatric Infectious Diseases, Department of Pediatrics, Hassenfeld Children's Hospital at New York University Langone Medical Center, New York, New York

${ }^{6}$ Department of Anesthesiology and Critical Care Medicine, New York University Langone Medical Center, New York, New York

7 Division of Pulmonary and Critical Care, Department of Internal Medicine, New York University Langone Medical Center, New York, New York
Address for correspondence Jenna S. Silverstein, MD, Department of Obstetrics and Gynecology, New York University Langone Medical Center, 550 First Avenue, NBV 9E2, New York, NY 10016 (e-mail: Jenna.Silverstein@nyulangone.org).

Am J Perinatol Rep 2020;10:e169-e175.

\begin{abstract}
Keywords

- COVID-19

- critical illness

- intubation

- pregnancy

- SARS-CoV-2

There is a current paucity of information about the obstetric and perinatal outcomes of pregnant novel coronavirus disease 2019 (COVID-19) patients in North America. Data from China suggest that pregnant women with COVID-19 have favorable maternal and neonatal outcomes, with rare cases of critical illness or respiratory compromise. However, we report two cases of pregnant women diagnosed with COVID-19 in the late preterm period admitted to tertiary care hospitals in New York City for respiratory indications. After presenting with mild symptoms, both quickly developed worsening respiratory distress requiring intubation, and both delivered preterm via caesarean delivery. These cases highlight the potential for rapid respiratory decompensation in pregnant COVID-19 patients and the maternal-fetal considerations in managing these cases.
\end{abstract}

The severe acute respiratory syndrome-coronavirus-2 (SARSCoV-2) is now widespread in the United States, with a particular concentration of cases in New York City, where community spread of disease is extensive. As of April 26, 2020, there were 153,204 confirmed cases in New York City, accounting for $17 \%$ of cases in the United States. ${ }^{1,2}$ There are limited data on pregnant women infected with SARS-CoV-2, though small case series from China report overall favorable obstetric and perinatal outcomes. Specifically, the World Health Organization (WHO)-China Joint Mission on novel coronavirus disease 2019 (COVID-19) reported that among 147 pregnant women with COVID-19, including 64 confirmed, received

April 27, 2020

accepted

April 30, 2020
DOI https://doi.org/

10.1055/s-0040-1712925. ISSN 2157-6998.
Copyright $\odot 2020$ by Thieme Medical Publishers, Inc., 333 Seventh Avenue, New York, NY 10001, USA. Tel: +1(212) 760-0888.
License terms

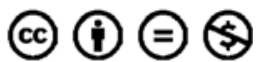


82 suspected, and 1 asymptomatic cases, $8 \%$ had severe disease and $1 \%$ were critical, with critical disease defined as respiratory failure requiring mechanical ventilation, shock, or other organ failure requiring intensive care. ${ }^{3}$ Other series from China have reported similar rates with no apparent increased risk of severe or critical illness for pregnant patients when compared with nonpregnant patients. ${ }^{4-6}$ These findings differ from the observed higher frequency of poor outcomes in pregnant women with Middle Eastern respiratory syndrome (MERS) and SARS. ${ }^{7}$

A case series of COVID-19 in pregnancy in the United States was recently reported, with two of seven patients becoming critically ill in the postpartum period. ${ }^{8}$ We report here two pregnant women with no medical comorbidities, one under 18 years of age, diagnosed with COVID-19 at 34 and 36 weeks of gestation, respectively, who rapidly decompensated and underwent caesarean delivery under general anesthesia followed by prolonged mechanical ventilation.

\section{Case Discussions}

\section{Case 1}

A 17-year-old Hispanic woman (gravida 1, para 0) at $36^{1 / 7}$ weeks of gestation with no comorbid conditions presented to the labor and delivery triage unit with a complaint of uterine contractions. On review of systems, she reported 2 days of sore throat and chills, but denied fever, cough, chest pain, or shortness of breath. Physical examination revealed oral body temperature $(\mathrm{T})$ of $37.4^{\circ} \mathrm{C}$, blood pressure (BP) of $101 / 51 \mathrm{~mm} \mathrm{Hg}$, pulse (HR) of 134 beats per minute (bpm), respiratory rate (RR) of 17 breaths per minute, and peripheral oxygen saturation $\left(\mathrm{SpO}_{2}\right)$ of $100 \%$. Electrocardiogram demonstrated sinus tachycardia. Fetal assessment was reassuring. Complete blood count demonstrated a normal white cell count ( - Table 1). After 2 hours of intravenous (IV) fluid administration her HR improved to 116 , contractions resolved, and she was discharged home.

Two days later she returned with complaints of chills and malaise, dry cough, chest pain, and shortness of breath. She was placed on droplet and contact precautions with concern for SARS-CoV-2 infection. Vital signs demonstrated T, $39.7^{\circ} \mathrm{C}$; BP, 97/60 mm Hg; HR, 145 bpm; RR, 30 breaths per minute; and $\mathrm{SpO}_{2}, 100 \%$. She was ill-appearing with bilateral conjunctival injection and flushed face, with clear lungs to auscultation, and nontender costovertebral angles, abdomen, and calves. Fetal nonstress test showed fetal tachycardia of $165 \mathrm{bpm}$ baseline but was otherwise reassuring. Laboratory evaluation showed mild anemia, mild thrombocytopenia, lymphopenia, and normal white cell count ( - Table 1 ). Specimens for respiratory pathogen testing including COVID-19 using New York SARS-CoV-2 real-time polymerase chain reaction (RT PCR) under Emergency Use Authorization (EUA) run by the department of health were collected. Chest radiograph revealed an opacity projecting over the left hilum concerning for consolidation (-Fig. 1A). She was admitted to the pediatric service with ongoing consultation by maternal fetal medicine and treated with IV fluids and acetaminophen. She was initiated on IV ceftriaxone 1 -g daily and oral azithromycin $500 \mathrm{mg}$ (followed by $250 \mathrm{mg}$ daily) for possible superimposed bacterial pneumonia on suspected SARS-CoV-2 viral pneumonia. She improved throughout the hospital day 2 and had no respiratory difficulty, with $\mathrm{SpO}_{2}>95 \%$ on room air. SARS-CoV-2 resulted positive, and testing for alternative pathogens was negative (-Table 1). Laboratories were significant for new leukopenia, worsening thrombocytopenia, and elevated C-reactive protein (-Table $\mathbf{1}$ ).

She continued to have intermittent fevers, and beginning in the evening of hospital day 3 her respiratory status began to decompensate. She denied dyspnea but vital signs were significant for $\mathrm{T}, 39.4^{\circ} \mathrm{C}$; BP, $88 / 44 \mathrm{~mm} \mathrm{Hg}$; pulse (HR), $136 \mathrm{bpm}$; RR, 52 breaths per minute; and $\mathrm{SpO}_{2}, 94 \%$ on room air. Repeat chest radiograph demonstrated increased patchy bilateral airspace opacities compatible with multifocal pneumonia (-Fig. 1B), therefore vancomycin $1 \mathrm{~g}$ every 12 hours and hydroxychloroquine $200 \mathrm{mg}$ orally in every 12 hours were initiated. In under 12 hours, she progressed from feeling comfortable on room air to requiring 2 L of oxygen via nasal cannula with a respiratory rate of 30 breaths per minute (achieving SpO2 = 95-97\%), and exhibited nasal flaring and increased work of breathing. She was evaluated by the critical care team who recommended transfer to the intensive care unit (ICU) for early intubation due to rapid respiratory decompensation (-Fig. 2). An interdisciplinary decision with critical care and maternal-fetal medicine teams was made to perform simultaneous intubation and cesarean delivery. The patient was transferred to a negative pressure operating room on labor and delivery (previously designated in 2014 during the Ebola outbreak), intubated with appropriate precautions for an aerosolizing procedure, and delivered by uncomplicated low transverse primary caesarean at $36^{6 / 7}$ weeks of gestation. She delivered a baby girl with Apgar's scores of 6 and 8 at 1 and 5 minutes, respectively, weighing $3,225 \mathrm{~g}$. The neonate was isolated and remained clinically well until discharge on day of life 2, and testing for SARS-CoV-2 resulted negative.

Postoperatively the patient was transferred to the ICU intubated. She was quickly weaned and extubated on postoperative day 0 , but necessitated reintubation and broadening of antibiotics on postoperative day 2 due to increased work of breathing requiring $8 \mathrm{~L}$ of oxygen via nasal cannula and worsening chest radiograph findings ( - Fig. 1C). She completed a 5-day course of hydroxychloroquine $200 \mathrm{mg}$ orally in every 12 hours and was given tocilizumab 400-mg IV once on postoperative day 4 . On postoperative day 6, her C-reactive protein began to decrease ( - Table $\mathbf{1}$ ) and on postoperative day 7 , she was successfully extubated. On postoperative day 11 (hospital day 15), she was discharged to home.

\section{Case 2}

A 34-year-old Caucasian woman (gravida 6, para 5) at $34^{0 / 7}$ weeks of gestation with pregnancy complicated by obesity (BMI $=40 \mathrm{~kg} / \mathrm{m}^{2}$ ) presented to the emergency department with a 5-day history of malaise and fever to $101^{\circ} \mathrm{F}$ at home, 2 days of dry cough and nausea, and 1 day of shortness of breath. Upon review of systems, she endorsed 1 week of bilateral lower extremity edema left greater than right, and decreased fetal movement. Vital signs showed $\mathrm{T}, 37.1^{\circ} \mathrm{C}$; 


\begin{tabular}{|c|c|c|c|c|c|c|c|c|c|c|c|c|c|c|c|c|c|c|c|c|c|c|c|c|c|c|c|}
\hline 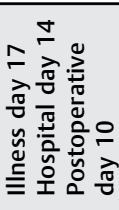 & $\stackrel{i}{\curvearrowright}$ & Fं & $\begin{array}{l}\underset{+}{q} \\
\end{array}$ & $\stackrel{\bar{\infty}}{+}$ & 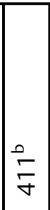 & \begin{tabular}{l}
0 \\
0 \\
0 \\
\hdashline
\end{tabular} & \begin{tabular}{|l|}
$\stackrel{g}{m}$ \\
$m$
\end{tabular} & $\underset{\Xi}{\ddagger}$ & 㒸 & 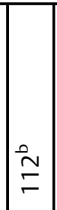 & $\underset{\infty}{\sim}$ & $\bar{\sim}$ & $=$ & ณ & $\stackrel{9}{0}$ & & & 1 & 1 & & 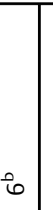 & 1 & & & & & \\
\hline 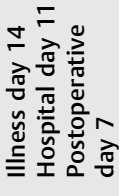 & $\stackrel{m}{r}$ & 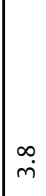 & $\underset{\sim}{\stackrel{q}{*}}$ & $\stackrel{m}{\stackrel{n}{r}}$ & $\begin{array}{l}\stackrel{0}{ \pm} \\
\stackrel{0}{m}\end{array}$ & \%ू. & $\mid \begin{array}{c}\bar{m} \\
\dot{m}\end{array}$ & 迺 & $\stackrel{\leftrightarrow}{\stackrel{\rho}{m}}$ & 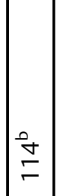 & 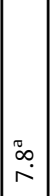 & $\stackrel{\sim}{\sim}$ & $\sigma$ & $\infty$ & : & $\stackrel{\sim}{\stackrel{n}{N}}$ & כ̋ & $\div$ & $\stackrel{\infty}{\sim}$ & $\stackrel{\tilde{m}}{\tilde{m}}$ & in & 1 & & & & & \\
\hline 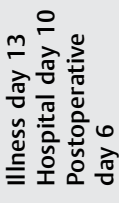 & $\stackrel{0}{\infty}$ & $\stackrel{g}{m}$ & $\stackrel{m}{n}$ & 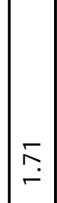 & $\begin{array}{l}\stackrel{?}{0} \\
\text { m }\end{array}$ & $\stackrel{0}{m} \stackrel{0}{\circ}$ & $\underset{m}{\stackrel{j}{i}}$ & 守 & 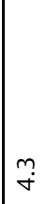 & $\stackrel{\stackrel{m}{m}}{\stackrel{m}{c}}$ & $\left|\begin{array}{l}0 \\
\infty\end{array}\right|$ & $\stackrel{\sim}{\sim}$ & $\circ$ & $\stackrel{\infty}{\circ}$ & ¿t. & 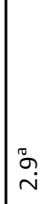 & $\tilde{o}$ & $\stackrel{\infty}{\sim}$ & న & 咛 & $\hat{\tilde{\hat{m}}}$ & 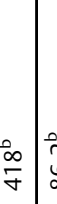 & 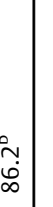 & & & & \\
\hline 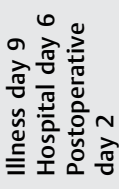 & م̂ & $\stackrel{\circ}{\stackrel{\leftrightarrow}{*}}$ & $\begin{array}{l}\hat{\hat{N}} \\
\infty\end{array}$ & 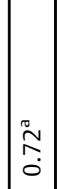 & $\stackrel{\mathcal{Z}}{\sim}$ & 邑 & $\underset{m}{\stackrel{j}{m}}$ & 古 & 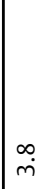 & : & $\mid \begin{array}{l}0 \\
\\
\end{array}$ & $\stackrel{\infty}{\infty}$ & $m$ & ڤ్రి & 占 & 品 & $\stackrel{+}{0}$ & $\tilde{\sim}$ & 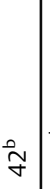 & $\stackrel{0}{\infty} \underset{\infty}{-}$ & $\stackrel{\infty}{\mathbb{N}}$ & $\stackrel{\stackrel{\leftrightarrow}{\sim}}{\sim}$ & & & 1 & & \\
\hline 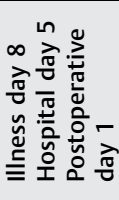 & ف & 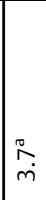 & m̊n & 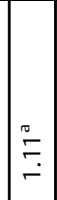 & 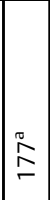 & $\stackrel{\circ}{\circ}$ & $\left|\begin{array}{c}n \\
\\
\bar{m}\end{array}\right|$ & 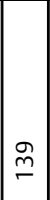 & $\stackrel{\leftrightarrow}{\text { re }}$ & 余 & 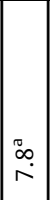 & $\approx$ & $a$ & $\pi$ & $\stackrel{\bullet}{0}$ & 吕 & $\tilde{c}$ & $\stackrel{\infty}{\sim}$ & $\stackrel{8}{\circ}$ & $\stackrel{\sim}{\bar{N}}$ & 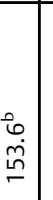 & 1 & & 1 & 1 & & \\
\hline 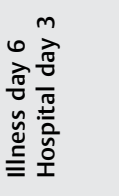 & نْ & $\stackrel{\substack{m \\
m}}{n}$ & $\underset{\sim}{\stackrel{\sim}{*}}$ & $\mid \begin{array}{l}0 \\
\hat{n} \\
0 \\
0\end{array}$ & $\mid \begin{array}{l}0 \\
0 \\
0 \\
0\end{array}$ & นัn & $\mid \begin{array}{c}\tilde{n} \\
\stackrel{\sim}{\sim}\end{array}$ & $\stackrel{\infty}{\sim}$ & مَn & 용 & 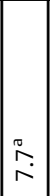 & $\stackrel{5}{\circ}$ & $\circ$ & $\stackrel{n}{\wedge}$ & 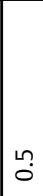 & 1 & 1 & 1 & 1 & & 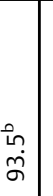 & 1 & I & 1 & 1 & & 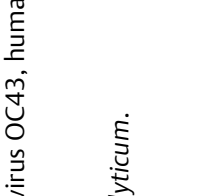 \\
\hline 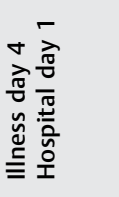 & भ่ & 幽 & $\mid \begin{array}{l}\infty \\
\dot{+}\end{array}$ & $\mid \begin{array}{l}\infty \\
0 \\
0 \\
0\end{array}$ & $\stackrel{\sigma}{\sigma}$ & مَn & $\mid \begin{array}{l}\sigma \\
\tilde{D} \\
\dot{2}\end{array}$ & $\stackrel{\substack{m \\
m}}{m}$ & $\stackrel{\leftrightarrow}{\text { ri }}$ & $\stackrel{\varrho}{\circ}$ & $\bar{\infty}$ & $\stackrel{5}{\circ}$ & $\sigma$ & $\approx$ & $\stackrel{\bullet}{0}$ & نَّ & $\stackrel{\nabla}{0}$ & $\simeq$ & $\stackrel{\sim}{\sim}$ & $\stackrel{\sim}{\stackrel{+}{\sim}}$ & 1 & 1 & & 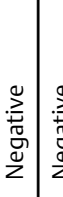 & 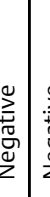 & 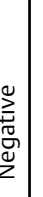 & 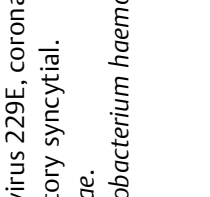 \\
\hline 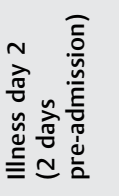 & : & 幽 & $\underset{\sim}{+}$ & $\mid \begin{array}{l}\tilde{\sigma} \\
\hat{\sigma} \\
0 \\
0\end{array}$ & $\begin{array}{l}\stackrel{0}{\circ} \\
\stackrel{\circ}{0}\end{array}$ & 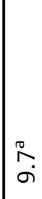 & $\begin{array}{l}\text { ஸ̃ } \\
\text { స̃. }\end{array}$ & 1 & 1 & 1 & 1 & 1 & 1 & 1 & 1 & 1 & 1 & 1 & 1 & 1 & 1 & 1 & 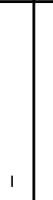 & 1 & & & 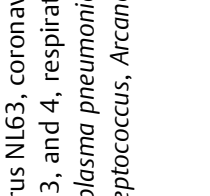 \\
\hline 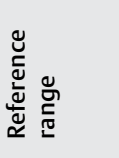 & 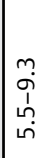 & $\begin{array}{l}0 \\
\dot{0} \\
\infty \\
\dot{m} \\
\dot{m}\end{array}$ & $\begin{array}{l}0 \\
0 \\
m \\
m\end{array}$ & 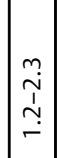 & $\mid \begin{array}{c}0 \\
0 \\
1 \\
\tilde{\alpha} \\
\sigma\end{array}$ & 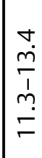 & $\mid \begin{array}{c}\hat{\infty} \\
0 \\
0 \\
1 \\
\dot{i} \\
m\end{array}$ & $\mid \begin{array}{c}\frac{1}{+} \\
\overline{1} \\
0 \\
m\end{array}$ & $\begin{array}{l}\sim \\
0 \\
\dot{\rho} \\
\dot{m} \\
\dot{m}\end{array}$ & $\mid$\begin{tabular}{l|}
0 \\
0 \\
$\vdots$ \\
$\vdots$ \\
$\infty$ \\
$o$
\end{tabular} & $\mid \begin{array}{l}0 \\
0 \\
0 \\
0 \\
\infty \\
\infty\end{array}$ & $\begin{array}{l}\tilde{m} \\
\tilde{n} \\
\tilde{n}\end{array}$ & $\begin{array}{l}\infty \\
\frac{1}{b}\end{array}$ & $\begin{array}{l}0 \\
\hat{P} \\
\hat{R}\end{array}$ & $\frac{F}{\frac{7}{1}}$ & 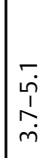 & 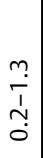 & $\begin{array}{c}\stackrel{n}{p} \\
\stackrel{1}{r} \\
\end{array}$ & $\begin{array}{l}\stackrel{m}{p} \\
\stackrel{\underline{F}}{2}\end{array}$ & $\begin{array}{l}8 \\
\vdots \\
\vdots \\
\\
\end{array}$ & $\begin{array}{l}n \\
\vdots \\
c\end{array}$ & 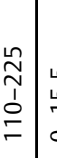 & 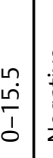 & 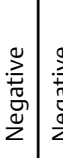 & & 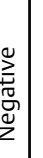 & 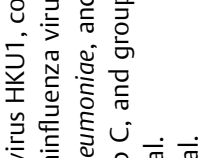 \\
\hline 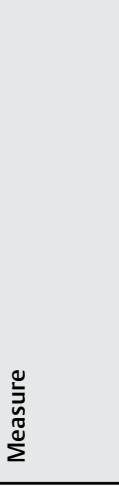 & 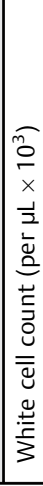 & 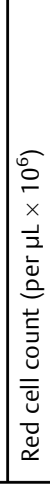 & 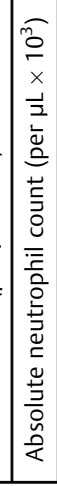 & 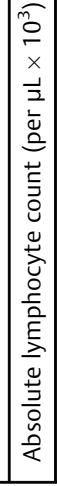 & 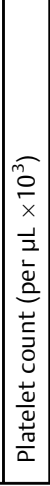 & 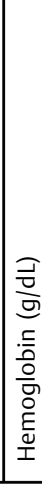 & 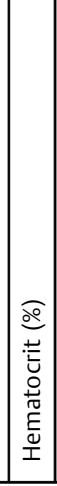 & 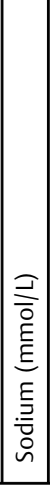 & 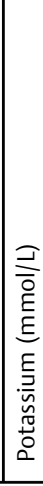 & 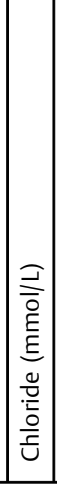 & 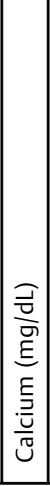 & 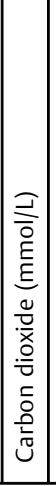 & 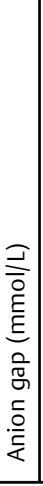 & 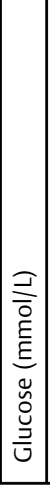 & 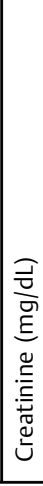 & 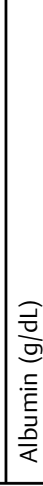 & 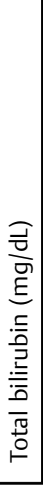 & 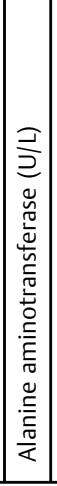 & 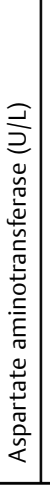 & 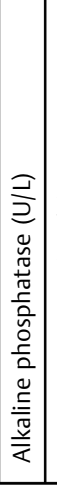 & 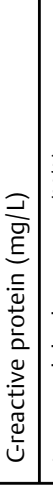 & 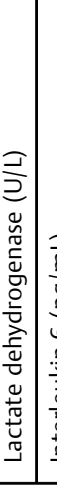 & 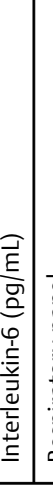 & 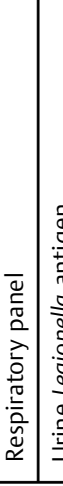 & 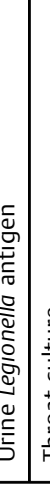 & 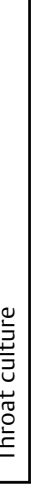 & 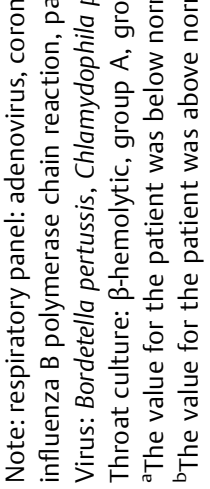 \\
\hline
\end{tabular}



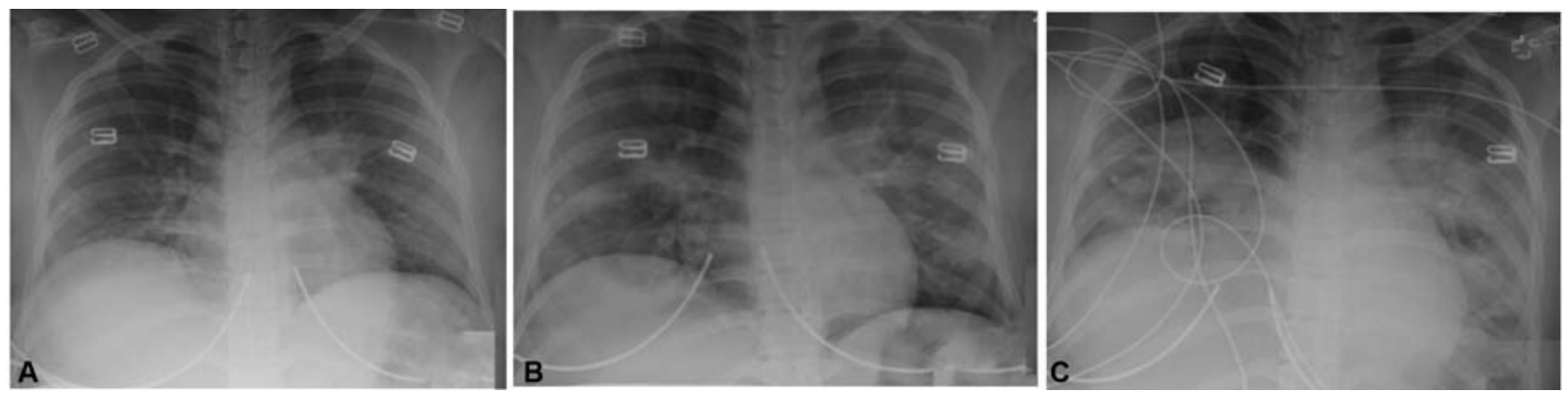

Fig. 1 Chest radiographs of Case 1. (A) Day of presentation to hospital for admission. (B) Hospital day 3, when new oxygen requirement began prompting intubation and delivery. (C) Hospital day 6/postoperative day 2, respiratory decompensation necessitating reintubation.

\section{Clinical Course}

Patient 1

Fig. 2 Time course of clinical events.

BP, 116/65 mm Hg; HR, 102 bpm; RR, 20 breaths per minute; and $\mathrm{SpO}_{2}, 97 \%$. She was well-appearing and in no respiratory distress, with bilateral diffusely coarse breath sounds noted. Workup was initiated for upper respiratory tract pathogens including COVID-19 using Cobas SARS-CoV-2 RT-PCR under EUA, which was tested within the hospital laboratory. Initial laboratory evaluation demonstrated mild thrombocytopenia, mildly elevated aspartate aminotransferase, and elevated C-reactive protein, with normal leukocytes, absolute lymphocyte count, and carbon dioxide ( - Table 2 ). Chest radiograph demonstrated patchy bilateral pulmonary and perihilar opacities with associated peribronchial thickening (-Fig. 3A). Lower extremity duplex ultrasound ruled out deep vein thrombosis, and electrocardiogram demonstrated sinus tachycardia. Fetal status was reassuring. She was admitted to the medicine service with ongoing consultation by maternal fetal medicine and initiated on azithromycin 500-mg IV daily and ceftriaxone 1-g IV daily (the latter due to her possible superimposed bacterial pneumonia seen on chest imaging).

In the morning of hospital day 2 , she was febrile to $38.8^{\circ} \mathrm{C}$ with $94 \% \mathrm{SpO}_{2}$ on room air with otherwise normal vitals, and she was given acetaminophen. A few hours later, she was afebrile but required $1 \mathrm{~L}$ of oxygen by nasal cannula to maintain $\mathrm{SpO}_{2}$ saturation of 92 to $94 \%$. Testing for alternative pathogens resulted negative (-Table 2 ) and SARS-CoV-2 PCR testing resulted positive. Blood cultures were collected which later resulted negative. By the afternoon, she became progressively tachypneic with RR, 36 breaths per minute, and soon thereafter required $6 \mathrm{~L}$ of oxygen by nasal cannula to maintain her $\mathrm{SpO}_{2}$ at $95 \%$ associated with increased work of breathing. Twenty hours after she initially developed an oxygen requirement, and just 5 hours after she required more than $1 \mathrm{~L}$ supplemental oxygen, she required intubation for respiratory support (-Fig. 2). After multidisciplinary discussion with critical care and maternal fetal medicine teams, simultaneous intubation and caesarean delivery was recommended, followed by transfer to the ICU.

The patient was taken to a negative pressure operating room, intubated, and delivered by uncomplicated low transverse primary caesarean at $34^{1 / 7}$ weeks of gestation. She delivered a baby girl with Apgar's scores of 8 and 9 at 1 and 5 minutes, respectively, weighing $2,110 \mathrm{~g}$. The neonate was isolated and transferred to neonatal intensive care unit (NICU) for prematurity and monitoring. Testing for SARSCoV-2 performed on the neonate on day of life 1 was negative.

On postoperative day 1 , the patient remained intubated and sedated in the ICU. She continued on azithromycin, and a 5-day course of hydroxychloroquine of $200 \mathrm{mg}$ orally twice daily was initiated. She had intermittent fevers throughout postoperative days 3 to 5 and required more ventilatory support and was given tocilizumab 400-mg IV once. The protocol to prone patients who are difficult to oxygenate could not be performed due to delirium, postoperative pain control, and difficulty achieving optimal sedation. On postoperative day 6 , a percutaneous tracheostomy was placed due to significant sedation requirements and lack of progress on the ventilator. After this, she required less sedation, was more alert, and able to participate in respiratory exercises. Repeat chest radiograph demonstrated improvement in the 


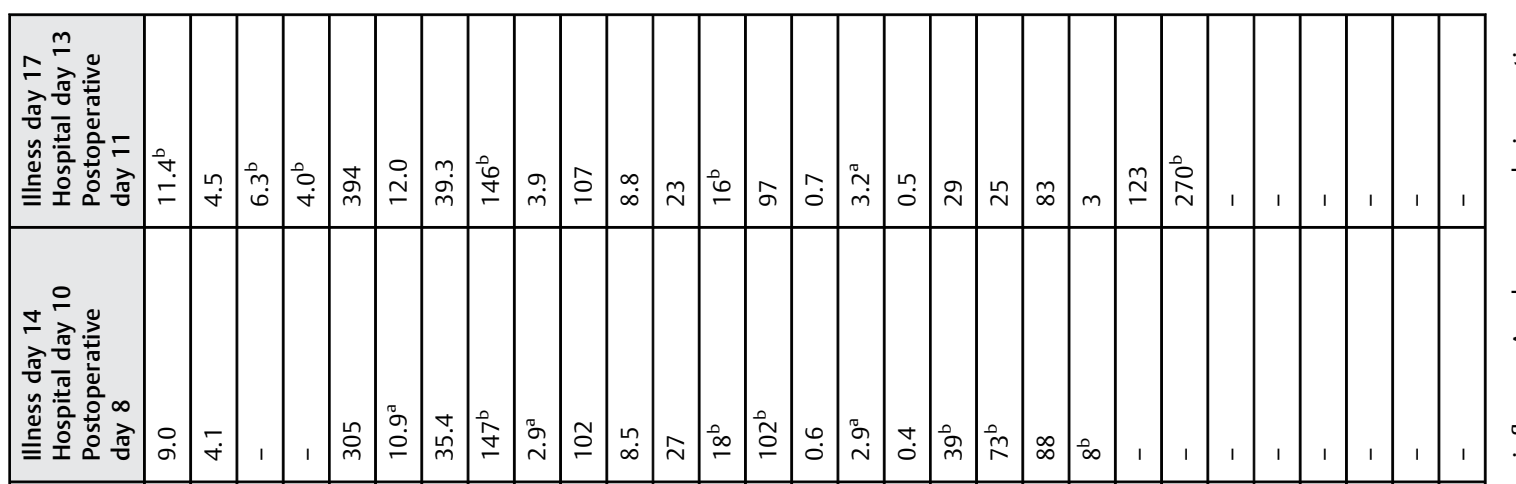

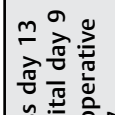

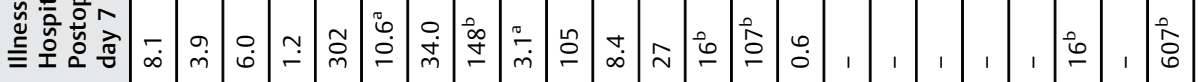

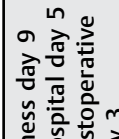

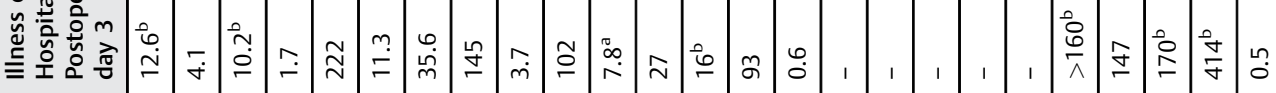

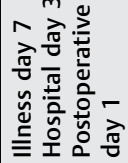

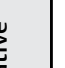



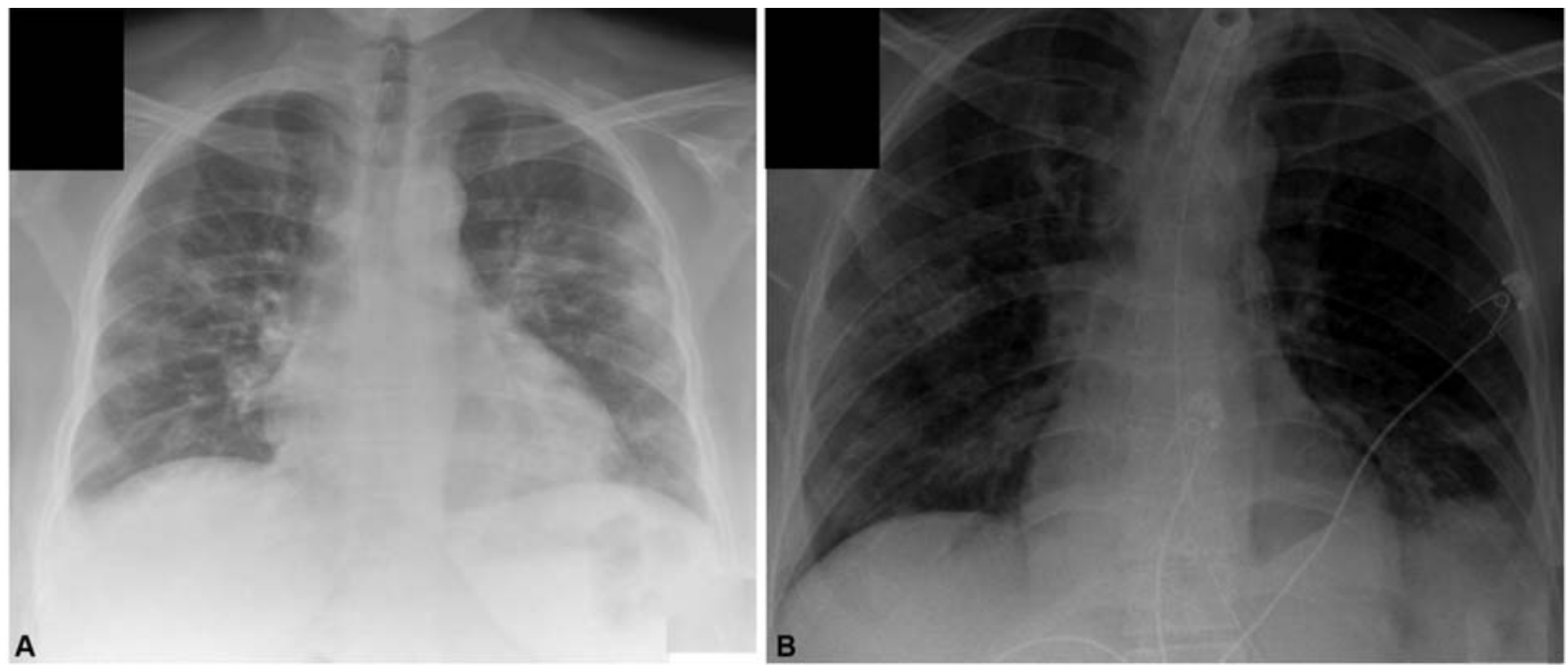

Fig. 3 Chest radiographs of case 2. (A) Day of presentation to hospital for admission. (B) Hospital day 8/postoperative day 6, repeat imaging before percutaneous tracheostomy.

bilateral patchy parenchymal opacities ( - Fig. 3B). On postoperative day 12 , she was transitioned to tracheostomy collar and transferred out of the ICU. After continuing her recovery and respiratory therapy for an additional week, her tracheostomy was decannulated on postoperative day 20 and she was discharged home on postoperative day 21 (hospital day 23).

\section{Discussion}

We report two cases of severe COVID-19 illness during pregnancy. These cases share several key elements that can be considered in the management of future pregnant COVID-19 patients. Both patients were under the age of 35 years with no significant comorbid conditions, and both were initially well-appearing with no oxygen requirement despite consolidations on chest radiograph. Within the first few days of hospital admission, they developed rapidly increasing oxygen requirements over the course of 6 to 12 hours, ultimately prompting need for mechanical ventilation and late preterm cesarean delivery followed by prolonged mechanical ventilation. Of note, in March 2020 when these patients were admitted, the approach to rapid respiratory decompensation was immediate intubation, which is now often delayed for a trial of noninvasive ventilation. Their course highlights the potential for swift respiratory decompensation in pregnant COVID-19 patients who develop even minimal oxygen requirements.

The risks and benefits of delivery in pregnant patients with critical respiratory illness from COVID-19 infections are not yet known, but prior experience with maternal acute respiratory distress syndrome (ARDS) and viral 2009/H1N1 influenza requiring mechanical ventilation in pregnancy reveals increased risk of fetal HR abnormalities, as well as fetal and neonatal mortality. ${ }^{9,10}$ Planned cesarean delivery therefore avoids the maternal and fetal risks of emergent delivery in a further decompensated or perimortem state. On the other hand, increased fluid administration and other physiologic changes associated with delivery may have a negative impact on the course of COVID-19 pneumonia. Ultimately, the decision to deliver in these two cases was also influenced by the favorable gestational age over 34 weeks but would be more challenging in pregnancies at an earlier gestational age. An interdisciplinary team should always be convened to weigh the unique medical, obstetric, and neonatal considerations for each case.

\section{Conclusion}

A recent publication from a nearby tertiary care center in New York City reported two of seven confirmed pregnant COVID-19 patients admitted for obstetric indications required ICU admission and critical care postpartum. ${ }^{8}$ Together, these cases suggest that young, otherwise healthy, pregnant patients with COVID-19 infections are at risk of rapid deterioration in pregnancy, as well as postpartum, requiring prolonged mechanical ventilation. These cases add to a growing body of literature about the natural history of severe COVID-19 disease in pregnancy and can be used to inform management and counseling of pregnant women hospitalized with complications related to COVID-19.

\section{Funding}

No financial support was received for this case report. IRB exemption status was received for this report.

Conflict of Interest

None declared.

\section{References}

1 WHO. Coronavirus disease 2019 (COVID-19) Situation Report-97. Available at: https://www.who.int/docs/default-source/coronaviruse/situation-reports/20200426-sitrep-97-covid-19.pdf? sfvrsn=d1c3e800_6. Accessed May 6, 2020

2 NYC Health. Coronavirus Disease 2019 (COVID-19) Daily Data Summary. Available at: https://www1.nyc.gov/site/doh/covid/ covid-19-data.page. Accessed May 6, 2020 
3 WHO. Report of the WHO-China Joint Mission on Coronavirus Disease 2019 (COVID-19). Available at: https://www.who.int/docs/default-source/coronaviruse/who-china-joint-missionon-covid-19-final-report-1100hr-28feb2020-11 mar-update. pdf?sfvrsn=1a13fda0_2\&download=true. Accessed may 6, 2020

4 Liu Y, Chen H, Tang K, Guo Y. Clinical manifestations and outcome of SARS-CoV-2 infection during pregnancy. J Infect 2020 (e-pub ahead of print) . Doi: 10.1016/j.jinf.2020.02.028

5 Chen H, Guo J, Wang C, et al. Clinical characteristics and intrauterine vertical transmission potential of COVID-19 infection in nine pregnant women: a retrospective review of medical records. Lancet 2020;395(10226):809-815

6 Yu N, Li W, Kang Q et al. Clinical features and obstetric and neonatal outcomes of pregnant patients with COVID-19 in Wuhan, China: a retrospective, single-centre, descriptive study. Lancet Infect Dis 2020 (e-pub ahead of print) . Doi: 10.1016/ S1473-3099(20)30176-6
7 Rasmussen SA, Smulian JC, Lednicky JA, Wen TS, Jamieson DJ. Coronavirus disease 2019 (COVID-19) and pregnancy: what obstetricians need to know. Am J Obstet Gynecol 2020;222(05): 415-426

8 Breslin NBC, Miller R, Fuchs K, Goffman D, Gyamfi-Bannerman C, D'Alton M. COVID-19 in pregnancy: early lessons. Am J Obstet Gynecol MFM 2020 (e-pub ahead of print) . Doi: 10.1016/j. ajogmf.2020.100111

9 Catanzarite V, Willms D, Wong D, Landers C, Cousins L, Schrimmer D. Acute respiratory distress syndrome in pregnancy and the puerperium: causes, courses, and outcomes. Obstet Gynecol 2001;97(5, Pt 1):760-764

10 ANZIC Influenza Investigators and Australasian Maternity Outcomes Surveillance System. Critical illness due to 2009 A/ H1N1 influenza in pregnant and postpartum women: population based cohort study. BMJ 2010;340:c1279 